Grand Valley State University

ScholarWorks@GVSU

$1-2012$

\title{
Product Angular Distributions in the Ultraviolet Photodissociation of $\mathrm{N}_{2} \mathrm{O}$
}

George C. McBane

Grand Valley State University, mcbaneg@gvsu.edu

Reinhard Schinke

Max-Planck-Institut für Dynamik und Selbstorganisation

Follow this and additional works at: https://scholarworks.gvsu.edu/chm_articles

Part of the Chemistry Commons

\section{ScholarWorks Citation}

McBane, George C. and Schinke, Reinhard, "Product Angular Distributions in the Ultraviolet Photodissociation of $\mathrm{N}_{2} \mathrm{O}$ " (2012). Peer Reviewed Articles. 2.

https://scholarworks.gvsu.edu/chm_articles/2

This Article is brought to you for free and open access by the Chemistry Department at ScholarWorks@GVSU. It has been accepted for inclusion in Peer Reviewed Articles by an authorized administrator of ScholarWorks@GVSU.

For more information, please contact scholarworks@gvsu.edu. 


\title{
Product angular distributions in the ultraviolet photodissociation of $\mathrm{N}_{2} \mathrm{O}$
}

\author{
George C. McBane ${ }^{1, a)}$ and Reinhard Schinke $\left.{ }^{2, b}\right)$ \\ ${ }^{1}$ Department of Chemistry, Grand Valley State University, Allendale, Michigan 49401, USA \\ ${ }^{2}$ Max-Planck-Institut für Dynamik und Selbstorganisation, D-37073 Göttingen, Germany
}

(Received 28 October 2011; accepted 4 January 2012; published online 30 January 2012)

\begin{abstract}
The angular distribution of products from the ultraviolet photodissociation of nitrous oxide yielding $\mathrm{O}\left({ }^{1} D\right)$ and $\mathrm{N}_{2}\left(X^{1} \Sigma_{g}^{+}\right)$was investigated using classical trajectory calculations. The calculations modeled absorption only to the $2^{1} A^{\prime}$ electronic state but used surface-hopping techniques to model nonadiabatic transitions to the ground electronic state late in the dissociation. Observed values of the anisotropy parameter $\beta$, which decrease as the product $\mathrm{N}_{2}$ rotational quantum number $j$ increases, could be well reproduced. The relatively low observed $\beta$ values arise principally from nonaxial recoil due to the very strong bending forces present in the excited state. In the main part of the product rotational distribution near $203 \mathrm{~nm}$, an unusual dynamical effect produces the decrease in $\beta$ with increasing $j$; nonaxial recoil effects remain approximately constant while higher $j$ product molecules arise from parent molecules that had their transition dipole moments aligned more closely along the molecular axis. In both low and high $j$ tails of the rotational distribution, the variations in $\beta$ with $j$ are caused by changes in the extent of nonaxial recoil. In the high- $j$ tail, additional torque present on the ground state potential energy surface following nonadiabatic transitions causes both the additional rotational excitation and the lower $\beta$ values. () 2012 American Institute of Physics. [doi:10.1063/1.3679171]
\end{abstract}

\section{INTRODUCTION}

Nitrous oxide, $\mathrm{N}_{2} \mathrm{O}$, is a trace atmospheric gas. Its destruction in the atmosphere is caused primarily by photodissociation through the first absorption band, which has a broad Gaussian shape centered near $180 \mathrm{~nm}$. Hopper ${ }^{1}$ published an influential theoretical study of the electronic structure of $\mathrm{N}_{2} \mathrm{O}$, and several later treatments have provided more detail. ${ }^{2-5}$ The first absorption band could have contributions from two different singlet electronic states in linear $\mathrm{N}_{2} \mathrm{O}$, the first ${ }^{1} \Sigma^{-}$and ${ }^{1} \Delta$ states. Transitions to both states are forbidden in the linear molecule. Away from linearity, the $1^{1} \Sigma^{-}$state becomes $1^{1} A^{\prime \prime}$ while the $1^{1} \Delta$ state splits into the two Renner-Teller components $2{ }^{1} A^{\prime}$ (the "A state") and $2{ }^{1} A^{\prime \prime}$. The ground, $1^{1} A^{\prime \prime}$, and $2^{1} A^{\prime}$ states all correlate adiabatically to $\mathrm{O}\left({ }^{1} D\right)$ $+\mathrm{N}_{2}\left(X^{1} \Sigma_{g}^{+}\right)$products while the $2^{1} A^{\prime \prime}$ state correlates to more highly excited states. The $1{ }^{1} A^{\prime \prime}$ and $2{ }^{1} A^{\prime}$ excited states are both strongly bent and consequently the $\mathrm{N}_{2}$ product is rotationally excited. The transition dipole moment calculations of both Daud et al. ${ }^{3}$ and Nanbu and Johnson ${ }^{4}$ indicate that absorption to $2{ }^{1} A^{\prime}$ should be stronger than that to $1^{1} A^{\prime \prime}$. The literature records a substantial effort to determine the relative contributions of the $1^{1} A^{\prime \prime}$ and $2^{1} A^{\prime}$ states to the absorption. ${ }^{3,6-10}$

In a series of recent papers, Schinke and collaborators have revisited the photodissociation of $\mathrm{N}_{2} \mathrm{O}$ using a new set of three-dimensional potential energy surfaces. ${ }^{5,11}$ The main conclusions from this work are that most of the experimentally observable properties associated with the first absorption band can be described satisfactorily on the basis of excitation

\footnotetext{
${ }^{a}$ Electronic mail: mcbaneg@gvsu.edu.

b) Electronic mail: rschink@gwdg.de.
}

of only the A state. These papers treated the absorption spectrum in detail, including its diffuse vibrational structure, ${ }^{5,11}$ its dependence on temperature, ${ }^{12}$ and its variation with isotopic composition. ${ }^{13}$ They also described product vibrational and rotational distributions computed both classically and quantum mechanically. ${ }^{14}$ The rotational distributions computed using only the $2{ }^{1} A^{\prime}$ excited state ended abruptly at a maximum value of $j$ near 80 that varied slightly with excitation wavelength. The experimental distributions, however, extend to somewhat higher $j$. Schinke and co-workers modeled nonadiabatic transitions between the initially excited A state and the ground $\mathrm{X}$ state using the fewest-switches trajectory surface hopping (TSH) method of Tully. ${ }^{15}$ The nonadiabatic coupling between the two surfaces is small immediately after excitation but becomes larger as the dissociation progresses. Rotational state distributions computed using the TSH model gave good agreement with experimental results in the high- $j$ tail of the rotational distribution.

In this paper we address the photodissociation anisotropy parameter $\beta$, another observable quantity that provides information about the detailed reaction mechanism. $\beta$ describes the correlation between the directions of the polarization vector of the exciting light and the outgoing velocity vectors of the products. It takes values between the limits of 2 , indicating velocities as nearly parallel to the polarization direction as possible, to -1 , indicating velocities as nearly perpendicular to the polarization as possible.

$\beta$ has been measured by several experimental groups at two wavelengths on the red side of the first absorption band. At $193 \mathrm{~nm}$, Felder et al. ${ }^{16}$ Springsteen et al.,${ }^{17}$ and Ahmed et al. ${ }^{18}$ all found that $\beta$ averaged over the whole product internal state distribution has a value near 0.5. For dissociation near $203 \mathrm{~nm}$, Neyer et al. ${ }^{19}$ and Kawamata et al. ${ }^{10}$ measured 
$\beta$ as a function of $\mathrm{N}_{2}$ final state. The two data sets are in excellent agreement with one another, and with several other less extensive reports. ${ }^{6,8,20}$ Both groups found that for $\mathrm{N}_{2}$ $(v=0), \beta$ declines slowly with increasing $j$ from $\beta \sim 1.0$ at the low end of the rotational distribution near $j=60$ to a value of about 0.7 at the peak of the rotational distribution near $j=78$. It then declines more steeply, reaching $\beta \sim 0$ at $j$ $=89$ near the high end of the rotational distribution. The work of Kawamata et al. ${ }^{10}$ and Teule et al. ${ }^{8}$ indicates that $\beta$ is not very sensitive to the initial bending excitation of $\mathrm{N}_{2} \mathrm{O}$, though the UV absorption cross section does increase substantially on vibrational excitation.

If the transition dipole vector lay along the $\mathrm{N}_{2} \mathrm{O}$ molecular axis and the $\mathrm{O}$ atom recoiled along the direction of the original $\mathrm{N}-\mathrm{O}$ bond, values of $\beta$ near the limit of 2 would be expected. Either a transition dipole directed away from the molecular axis (changing from a "parallel" toward a "perpendicular" transition) or a deviation of the outgoing velocity vector from the molecular axis ("nonaxial recoil") can reduce the observed values of $\beta$. Absorption to the A state $\left(2{ }^{1} A^{\prime}\right)$, whose transition dipole must lie in the triatomic plane, could therefore produce any value of $\beta$; the observed values would depend on the orientation of the transition dipole and the extent of nonaxial recoil. Absorption to the $1^{1} A^{\prime \prime}$ state would necessarily produce $\beta=-1$ in the absence of parent rotation, because the transition dipole is perpendicular to the triatomic plane.

In this paper, using a classical treatment including surface hopping, we show that the observed $\beta$ values are consistent with absorption purely to the A state and that nonaxial recoil, caused largely by strong bending forces on the excited state, is the main factor that controls the observed values of $\beta$. In addition, we identify two detailed features that describe the variation of $\beta$ across the rotational distribution. First, the slow decline of $\beta$ in the main part of the rotational distribution from $203 \mathrm{~nm}$ dissociation is not caused primarily by variation in the extent of nonaxial recoil but instead by variations in the direction of the $\mathrm{X}-\mathrm{A}$ transition dipole $\boldsymbol{\mu}_{\mathrm{XA}}$ between the subsets of absorbing molecules that produce different final $j$. Second, we show that the steep decline in $\beta$ beyond the maximum of the rotational distribution does arise from an increase in nonaxial recoil, brought on by the stronger torques present in the "isomerization pathway" on the ground state surface. Nonadiabatic effects in the exit channel of the photodissociation are therefore reflected in both the rotational distribution and in the corresponding anisotropy parameters $\beta(j)$.

Several experimental groups have also measured the angular momentum alignment of the $\mathrm{O}\left({ }^{1} D\right)$ fragment, that is, the spatial distribution of the electronic orbital angular momentum vector. $7,8,18,21,22$ Our classical, $J=0$ model is not well suited to treating those observations, and we do not address them here.

\section{COMPUTATIONAL METHODS}

We modeled the photodissociation using classical trajectories with total angular momentum $J=0$ and the fewest-switches surface hopping method ${ }^{15}$ as described in Ref. 14, using the adiabatic potential surfaces described in Ref. 5. The surfaces were computed with the aug-ccpVQZ basis $^{23}$ at the complete active space self-consistent field/multiconfiguration reference internally contracted configuration interaction level ${ }^{24-27}$ using the MOLPRO (Ref. 28) program suite and evaluated using three-dimensional splines. The calculations were done in Jacobi coordinates $R$ (distance from $\mathrm{O}$ atom to $\mathrm{N}_{2}$ center of mass), $r$ (N-N distance), and $\gamma$, the angle between the $R$ and $r$ line segments.

Initial conditions for the trajectories were selected using importance sampling. ${ }^{29}$ Coordinates and momenta were initially sampled from Gaussian distributions chosen to model the ground state vibrational wave function of $\mathrm{N}_{2} \mathrm{O}$ and candidate starting conditions whose energies were more than $0.005 \mathrm{eV}$ away from the desired total energy were discarded. After the set of trajectories had been completed, final $\mathrm{N}_{2}$ quantum numbers were assigned with the method described by Schmidt et al. ${ }^{14}$ Final state properties were computed with weighted averages over the trajectory set, using weights of $\left|\psi_{000}\right|^{2}\left|\boldsymbol{\mu}_{\mathrm{XA}}\right|^{2}\left|\sin \gamma_{0}\right| / g$ evaluated at $\left(R_{0}, r_{0}, \gamma_{0}\right)$, where $\psi_{000}$ is the ground state vibrational wavefunction of $\mathrm{N}_{2} \mathrm{O}$ and $g$ is the probability density function of the initial Gaussian coordinate distribution. We used 100000 trajectories at each total energy.

In the surface hopping calculations, only the $\gamma$ component of the nonadiabatic coupling was included, as described by Schmidt et al. $;^{14}$ their figure 10 shows the nonadiabatic matrix element $D_{\gamma}$ as a function of $\gamma$ for several values of $R$. We tested the influence of the $R$ component using nonadiabatic coupling matrix elements computed on a twodimensional grid with $r$ fixed at $2.13 a_{0}$, and found that in the region important for the product state dynamics the $R$ coupling was roughly ten times smaller than the $\gamma$ coupling.

During the surface hopping calculations, an adjustment must be made to the momenta at each hop in order to conserve total energy. It is possible that this adjustment, a somewhat artificial feature of the TSH method, might perturb the trajectories enough to invalidate the $\beta$ calculation. We tested two procedures for this adjustment. With the "democratic prescription" used by Schmidt et al. ${ }^{14}$ for $\mathrm{N}_{2} \mathrm{O}$ photodissociation and by us in an earlier ozone study, ${ }^{30}$ the necessary kinetic energy changes at surface hops are divided equally among the three Jacobi momenta. With the " $g$ prescription,"31,32 the momenta are adjusted at a hop along the direction given by the gradient of the potential difference between the initial and final electronic states. Because the excited and ground state surfaces diverge strongly in angle, with the $\boldsymbol{g}$ prescription a large fraction of the kinetic energy change at each hop is made in the rotational coordinate. The shape of the high- $j$ tail in the rotational distribution obtained by the democratic prescription was very similar to that we obtained in test calculations that made the hop to the $\mathrm{X}$ state without any momentum adjustment at all. In addition, with the democratic prescription the changes in $j$ at hops were usually small compared to subsequent changes due to potential surface anisotropy. The $g$ prescription, in contrast, gave a broader rotational distribution tail with a maximum at $j=90$, and the $\Delta j$ it required were often comparable to those produced during the post-hop dynamics. For this study, we found the democratic prescription preferable. 


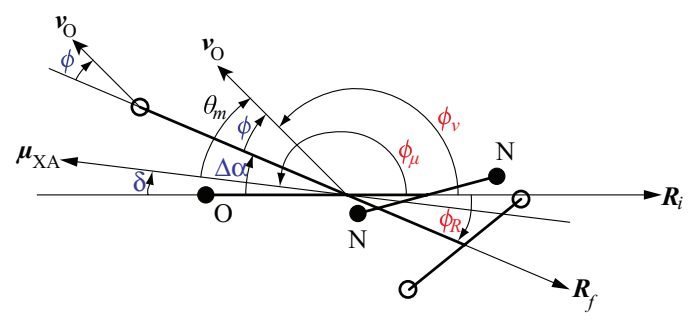

FIG. 1. Angles used in the present paper and in the empirical models of Demyanenko et al. ${ }^{35}$ and Brouard et al. ${ }^{36}$ The initial positions of the atoms are shown by solid dots; the positions later in the dissociation, after the torques have become negligible, by open circles. Angles used in the present treatment other than $\theta_{m}$ are defined in red on the right side of the diagram; they are measured counterclockwise, so that $\phi_{R}$ and $\theta_{m}$ have negative values. Angles used in the empirical models are defined in blue on the left side of the diagram, and are positive in the clockwise direction. The final rotational angular momentum vector of $\mathrm{N}_{2}$ extends upward out of the page. In Ref. 36, the angle $\phi$ is called $\alpha^{\prime}$ and $\Delta \alpha$ is assumed zero. $\theta_{m}$ is the same in our system and the empirical models except for sign.

In a classical treatment, ${ }^{33} \beta$ can be computed as an average over trajectories of $2 P_{2}\left(\cos \theta_{m}\right)$, where $\theta_{m}$ is the angle between the final velocity vector of the $\mathrm{O}$ atom and the direction of the transition dipole moment, $\boldsymbol{\mu}_{\mathrm{XA}}$, at the moment of excitation and $P_{2}(x)$ is a Legendre polynomial. We computed $\beta$ according to the procedure described in Ref. 34. We chose the space-fixed $z$ axis to lie along the initial $\boldsymbol{R}$ vector, pointing from $\mathrm{O}$ toward $\mathrm{N}_{2}$. Our coordinates were defined so that the terminal $\mathrm{N}$ atom (atom 1 ) had positive $y$ and $z$ coordinates for $\gamma>0$. For each trajectory, we evaluated the orientation angles of the transition dipole moment, $\phi_{\mu}$, and final $\mathrm{O}$ atom velocity, $\phi_{v}$, in a space-fixed frame and obtained $\theta_{m}=\phi_{v}-\phi_{\mu}$. We then used a weighted average, with weights as described above, to compute $\beta=\left\langle 2 P_{2}\left(\cos \theta_{m}\right)\right\rangle$. The relevant angles are indicated in red in Figure 1.

We evaluated the components of the transition dipole moment $\boldsymbol{\mu}_{\mathrm{XA}}$ using spline interpolation in 3-dimensional tables of $\mu_{y}$ and $\mu_{z}$ computed at the same level of theory as the potential..$^{5}$ The transition moment components, tabulated in the principal axis frame used by the MOLPRO electronic structure package, were transformed to the space-fixed system and the transition moment orientation angle in the range $\left[-\frac{\pi}{2}, \frac{\pi}{2}\right]$ was obtained with $\phi_{\mu}=\tan ^{-1}\left(\mu_{y} / \mu_{z}\right)$.

The components $v_{y}$ and $v_{z}$ of the final $\mathrm{O}$ atom velocity were obtained as described in Ref. 34, using the final orientation angle $\phi_{R}$ of the $R$ vector obtained through angular momentum constraints ${ }^{35}$ propagated along the trajectory. No special procedures were needed to compute $\beta$ from the surface hopping trajectories, except to check for possible artifacts as described below.

\section{RESULTS}

We first present the overall computational results and comparisons to experiment, and then offer more detailed analysis and physical interpretations.

The $j$-dependent angular distributions of products from $203 \mathrm{~nm}$ photodissociation have been measured by Neyer et $a l .{ }^{19}$ and Kawamata et al. ${ }^{9,10}$ The experiments used a single laser to photodissociate $\mathrm{N}_{2} \mathrm{O}$ and perform state-selective
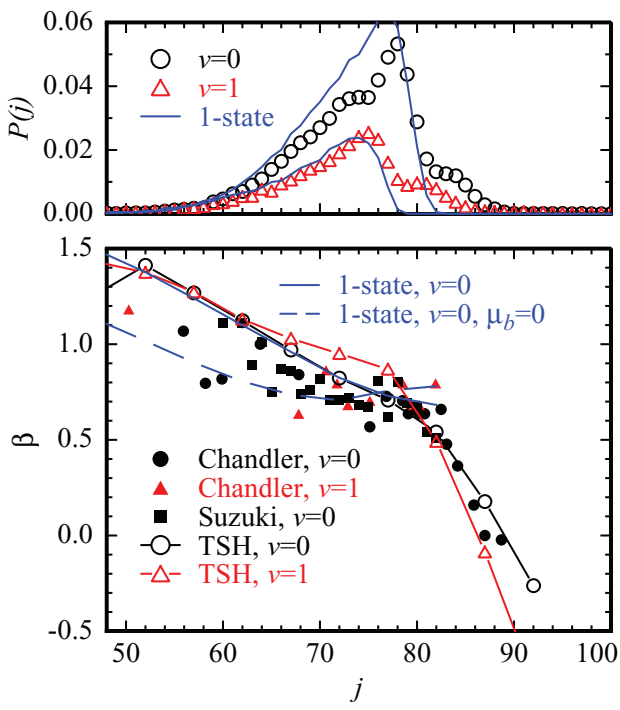

FIG. 2. The lower panel shows experimental and computed values of $\beta$ as a function of $j$ for photodissociation at $203.25 \mathrm{~nm}$. The computed $\beta$ values were averaged over rotational bins five units wide and plotted against the $j$ values at the center of each bin. Computed values from two-state surface hopping calculations are given as open symbols connected by lines, and those from one-state calculations as smooth lines. The dashed line shows results from a special calculation that forced the transition dipole moment to lie along the $a$ principal axis. The experimental points labeled "Chandler" are from Ref. 19; those labeled "Suzuki" are from Ref. 10. The upper panel shows computed rotational distributions for $v=0$ and 1 from one- and two-state calculations as reported in Ref. 14 .

$2+1$ photoionization of product $\mathrm{N}_{2}$. The $\mathrm{N}_{2}^{+}$ions were then detected by velocity mapping. An earlier measurement by Shafer et al., ${ }^{37}$ using Doppler-resolved $2+1$ multiphoton ionization of $\mathrm{O}\left({ }^{1} D\right)$ near $205.5 \mathrm{~nm}$, indicated only that $\beta>0$, though the authors recommended the limiting value $\beta=2$ on theoretical grounds and later investigators ${ }^{6,17}$ disagreed with that recommendation. Hanisco and Kummel, ${ }^{6}$ resolving the flight times of the $\mathrm{N}_{2}^{+}$ions in their $2+1$ REMPI measurement, also concluded that $\beta>0$.

Figure 2 shows the experimental data from the finalstate-resolved measurements ${ }^{10,19}$ and the corresponding results from the TSH calculations. Both experimental data sets include data for the dominant $v=0$ channel and they are in good agreement. The computed $\beta(j)$ match the experimental results remarkably well, capturing both the slower decline for $j \leq 80$ and the steeper decline at higher $j$. Only Neyer et al. ${ }^{19}$ reported data for $v=1$. The computed $\beta$ for $v=1$ are slightly too high, and the sparser experimental data do not extend into the high-j region, but the basic agreement is again very good.

We tested the effect of bending excitation of the parent with a calculation that weighted trajectories using the vibrational wave function of the $\mathrm{N}_{2} \mathrm{O}(0,1,0)$ state rather than $(0,0,0)$. We found that the effect on $\beta$ was very small, in agreement with the experimental evidence. ${ }^{8,10}$

Felder et al., ${ }^{16}$ using time-of-flight techniques with mass spectrometric detection, and Springsteen et al. ${ }^{17}$ using Doppler-resolved laser induced fluorescence detection of the $\mathrm{O}\left({ }^{1} D\right)$ product, measured $\beta$ averaged over the entire $\mathrm{N}_{2}$ final state distribution for dissociation at $193 \mathrm{~nm}$. The two results agree well, Felder et al. obtaining $\beta=0.48 \pm 0.02$ 

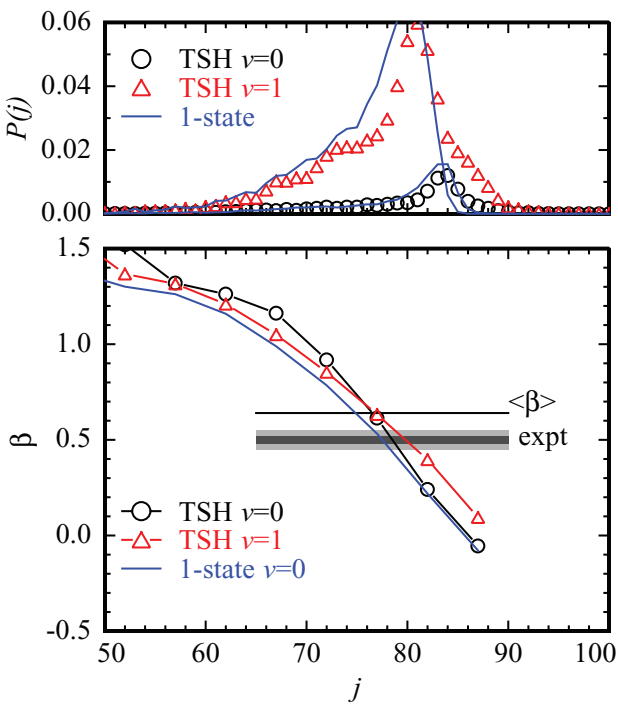

FIG. 3. $\beta$ for photodissociation at $193 \mathrm{~nm}$. The gray bands show the experimental ranges of Felder et al. (Ref. 16) (inner darker band) and Springsteen et al. (Ref. 17) (outer lighter band); these measurements represent averages over the entire final state distribution of $\mathrm{N}_{2}$, and are drawn between $j=65$ and $j=90$ to indicate the most important section of the final state distributions. The computed $\beta$ averaged over all final states is indicated with a horizontal line. In the lower panel, the computed one-state values for $v=1$ (not shown) agree almost exactly with the TSH values within the one-state rotational distribution. The upper panel shows computed rotational distributions for $v=0$ and 1 from one- and two-state calculations as reported in Ref. 14.

and Springsteen et al. obtaining $\beta=0.50 \pm 0.05$. Ahmed et al. ${ }^{18}$ using $\mathrm{O}\left({ }^{1} D\right)$ imaging, obtained $\beta=0.49$.

Figure 3 shows our computed $\beta(j)$ for 193 photodissociation, along with the corresponding computed final state distribution and the experimental values. The computed $\beta$ values show a smooth decline with increasing $j$. The average $\beta$ over the entire final state distribution is 0.64 , modestly higher than the experimental result.

\section{DISCUSSION}

\section{A. Average values of $\beta$}

In the main part of the rotational distributions below the maximum, one-state calculations and two-state TSH calculations give very similar results, as shown for the $v=0$ products of the $203.25 \mathrm{~nm}$ photodissociation in Figure 2. The dissociations producing those fragments remain on the A state during the part of the trajectory when significant forces are acting. The corresponding trajectories are all very similar. In onestate calculations at $203.25 \mathrm{~nm}$, the distribution of $\phi_{v}$ across the full range of final states is very narrow, with a peak at $135^{\circ}$ and a full width at half maximum of only $3^{\circ}$. (An $\mathrm{O}$ atom that departed directly along the initial $\mathrm{N}-\mathrm{O}$ bond from a linear initial configuration would have $\phi_{v}=180^{\circ}$.)

The distribution of $\phi_{\mu}$, the orientation angle of the transition dipole moment, is somewhat broader, extending from $168^{\circ}$ to nearly $180^{\circ}$; its maximum is at $173^{\circ}$. A trajectory with $\phi_{\mu}=173^{\circ}$ and $\phi_{v}=135^{\circ}$ would yield $\beta=0.86$, close to the average value of 0.90 produced by the one-state calculations at $203.25 \mathrm{~nm}$. We conclude that the observed average values of $\beta$, substantially lower than the limiting value of 2 that would be expected for axial recoil from a linear initial configuration, appear largely because of nonaxial recoil due to the strong bending forces in the excited state. (We use the term "nonaxial recoil" to indicate final velocities that are not parallel to the initial direction of the breaking bond, rather than adopting the usage of Demyanenko et al. ${ }^{35}$ who apply it to velocities that are not parallel to the $\boldsymbol{R}$ vector late in the dissociation.)

The transition dipole moment $\boldsymbol{\mu}_{\mathrm{XA}}$ is usually displaced from the initial $\boldsymbol{R}$ vector in the same direction as the final $\mathrm{O}$ atom velocity vector; this displacement reduces $\theta_{m}$. The average values of $\beta$ are therefore slightly larger than they would be if $\boldsymbol{\mu}_{\mathrm{XA}}$ was always parallel to $\boldsymbol{R}_{i}$.

\section{B. $\boldsymbol{j}$ dependence at lower $\boldsymbol{j}$}

We now consider the variation of $\beta$ with $j$ in the main part of the rotational distribution below its maximum. Figure 4 shows average values of the velocity and transition dipole orientation angles, $\phi_{v}$ and $\phi_{\mu}$, as functions of $j$. A reasonable expectation would be that an increase in final $j$ would represent an increase in bending forces, and therefore correlate with a change in $\phi_{v}$ further away from axial recoil. That is the idea embedded in the two-parameter empirical model applied to both OCS and $\mathrm{N}_{2} \mathrm{O}$ by Brouard et al., ${ }^{36}$ and it does apply in a general way across the entire rotational distribution. However, in the central section of the product distribution, 65 $\leq j \leq 80$, we find different behavior. Figure 4 shows that in our $203 \mathrm{~nm}$ trajectories, $\phi_{v}$ changes very little with final rotational state in this region. Instead, we find that as $j$ increases, $\phi_{v}$ remains constant but $\phi_{\mu}$ increases. In other words, trajectories that produce larger $j$ arise from molecules whose transition dipole moments lie closer to the initial $\boldsymbol{R}$ vector.

Figure 5 demonstrates this correlation. It shows contours of the "excitation functions" for $j, \beta=2 P_{2}\left(\cos \theta_{m}\right)$ $=3 \cos ^{2}\left(\phi_{v}-\phi_{\mu}\right)-1, \phi_{v}$, and $\phi_{\mu}$, as functions of initial coordinates $r_{0}$ and $\gamma_{0}$. These contours were generated from a special set of trajectories that were started with no initial momentum and with $R_{0}$ selected at each $\left(r_{0}, \gamma_{0}\right)$ pair to give a total energy corresponding to $204 \mathrm{~nm}$ excitation. Similar excitation functions for $j$ and the vibrational quantum number

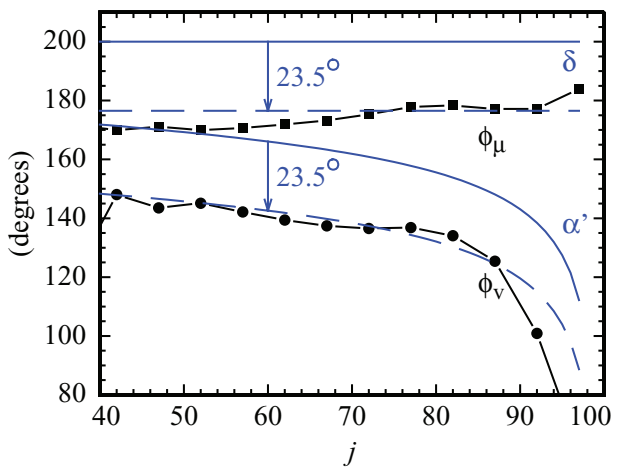

FIG. 4. Average values of the product exit angle, $\phi_{v}$, and of the transition dipole orientation angle, $\phi_{\mu}$, as functions of product $j$, averaged across $j$ bins as in Figure 2, from two-state calculations at $203.25 \mathrm{~nm}$. The smooth curves show versions of the empirical model used by Brouard et al. (Ref. 36). 


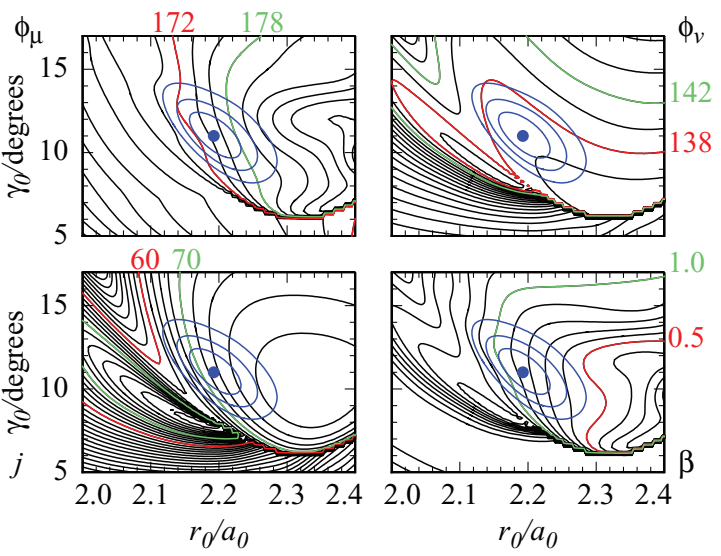

FIG. 5. Contours of velocity orientation angle $\phi_{v}$, transition dipole orientation angle $\phi_{\mu}$, final classical $\mathrm{N}_{2}$ angular momentum $j$, and the "singletrajectory anisotropy parameter" $\beta=2 P_{2}\left(\cos \theta_{m}\right)$. All were computed from trajectories on the A state started with no initial momentum and with $R_{0}$ selected at each $\left(r_{0}, \gamma_{0}\right)$ to give total energy corresponding to $204 \mathrm{~nm}$ excitation. The contour interval is $2^{\circ}$ for $\phi_{v}$ and $\phi_{\mu}, 2 \hbar$ for $j$, and 0.1 for $\beta$, with values at specific contours as indicated. The values of $\phi_{\mu}$ have been mapped onto $\left[0^{\circ}, 360^{\circ}\right]$ for ease of interpretation. The initial state weighting function, $|\psi|^{2}\left|\boldsymbol{\mu}_{\mathrm{XA}}\right|^{2}\left|\sin \gamma_{0}\right|$, is shown in blue, with the position of its maximum indicated by a dot and contours drawn at $75 \%, 50 \%$, and $25 \%$ of the maximum value.

were shown by Schmidt et al. ${ }^{14}$ In each panel, the weighting function for initial coordinate sampling is also shown, with its maximum (the "Franck-Condon point") indicated with a dot and with contours showing $75 \%, 50 \%$, and $25 \%$ relative weights.

In the lower left, the excitation contours for $j$ are displayed. The Franck-Condon region lies on the left (small $r_{0}$ ) side of a broad maximum that appears near $r_{0}=2.34 a_{0}, \gamma_{0}$ $=10.5^{\circ}$. As $r_{0}$ decreases across the Franck-Condon region, the final $j$ decreases steadily. $j$ also decreases with decreasing $\gamma_{0}$, though not as strongly.

The upper right panel shows that very little variation in $\phi_{v}$ should be expected across the Franck-Condon region; the outermost weight contour lies mostly between the $\phi_{v}$ $=136^{\circ}$ and $140^{\circ}$ contours. This observation is consistent with the very narrow distribution of $\phi_{v}$ generated in the trajectory calculations.

The upper left panel, showing the dependence of the transition dipole orientation angle $\phi_{\mu}$ on the initial geometry, indicates that $\phi_{\mu}$ is a relatively steep function of $r_{0}$ across the Franck-Condon region. Molecules that have larger $r_{0}$ also tend to have larger $\phi_{\mu}$; roughly, a $0.02 a_{0}$ change in $r_{0}$ produces a $2^{\circ}$ increase in $\phi_{\mu}$. This correlation reflects the coordinate dependence of the components of the transition dipole moment. The component perpendicular to the $a$ principal axis is small, usually less than 0.01 a.u., everywhere in the FranckCondon region and passes through zero for some geometries. The transition dipole direction does not depend very strongly on $\gamma_{0}$.

The lower right panel displays excitation function contours for $\beta$. Since $\phi_{v}$ changes very little across the FranckCondon region, the contours of $\beta$ mimic those of $\phi_{\mu}$ closely. As $r_{0}$ increases, $\phi_{\mu}$ also increases, so that the angle between $\boldsymbol{v}$ and $\boldsymbol{\mu}_{\mathrm{XA}}$ increases and $\beta$ decreases.
The decrease in the observed $\beta$ with final $j$ in the main part of the rotational distribution, therefore, does not come from a change in the extent of nonaxial recoil (a change in $\phi_{v}$ ). Instead, it arises because of the mutual dependence of $j$ and $\phi_{\mu}$ on $r_{0}$, the initial $\mathrm{N}_{2}$ bond length. All absorbing molecules are at least slightly bent, since the transition is forbidden in the linear molecule, and after excitation the angular potential on the excited state forces the bond angle to decrease. The resulting outgoing velocity $\boldsymbol{v}$ is about $45^{\circ}$ away from the $\boldsymbol{R}_{0}$ vector (that is, $\phi_{v} \approx 135^{\circ}$ ) for all dissociations. Molecules with smaller $r_{0}$ yield lower $j$ and usually have transition dipoles oriented away from $\boldsymbol{R}_{0}$ by a few degrees in the direction of $\boldsymbol{v}$. Molecules with larger $r_{0}$ produce higher $j$, and also tend to have initial dipole moments that lie closer to $\boldsymbol{R}_{0}$, farther from $\boldsymbol{v}$, and therefore produce lower $\beta$. The decrease in $\beta$ with increasing $j$ is therefore an effect of selective probing of different subsets of the excited molecules, rather than different post-excitation dynamics.

As a test of our assertion that variation in $\phi_{\mu}$ is the main cause of the variation in $\beta$ with $j$, we reanalyzed the same set of one-state trajectories while artificially forcing the transition dipole to lie along the $a$ principal axis of the molecule, near the $\boldsymbol{R}_{0}$ vector. The resulting $\beta(j)$ curve is shown as a dashed blue line in Figure 2. Within the main part of the rotational distribution where $65 \leq j \leq 80$, it is nearly constant near $\beta=0.8$, consistent with our description. In the low- $j$ tail where $j<65, \beta$ decreases with increasing $j$. This behavior is also described by Figure 5; in the part of the Franck-Condon region yielding the lowest $j$ values, near the upper left in the diagrams, the $\phi_{\mu}$ surface becomes flatter while the $\phi_{v}$ surface becomes steeper. In the low- $j$ tail of the distribution, the decline in $\beta$ with $j$ is indeed due to changes in the exit directions of the $\mathrm{O}$ atoms.

\section{C. $j$ dependence in the high $j$ tail}

We now consider the dependence of $\beta$ on $j$ above the maximum in the rotational distribution. Rotational distributions from the one-state calculations fall very abruptly beyond the maximum; they are shown by solid lines in the upper panels of Figures 2 and 3. Suzuki et al. assigned the high-j part of the rotational distribution from photodissociation of OCS to nonadiabatic transitions from the $\mathrm{A}$ to the $\mathrm{X}$ state in the exit channel. ${ }^{38}$ Following their lead, Schmidt et al. ${ }^{14}$ showed that nonadiabatic transitions from the $\mathrm{A}$ to the $\mathrm{X}$ state in the exit channel of the $\mathrm{N}_{2} \mathrm{O}$ dissociation, modeled with surface hopping, could explain the tail of the rotational distribution extending to higher $j$.

We examined the characteristics of the first surface hops for trajectories ending with $j \geq 80$ to make sure that our interpretation reflected dynamics on the two surfaces rather than artifacts introduced by the hopping procedure. For dissociation at $203.25 \mathrm{~nm}$, the total energy is $6.3961 \mathrm{eV}$, corresponding to a maximum product $j$ of about 103 . $68 \%$ of the first hops involve a decrease in potential energy less than $0.2 \mathrm{eV}$, $15 \%$ between 0.2 and $0.3 \mathrm{eV}$, and $14.6 \%$ between 0.3 and 0.7 $\mathrm{eV}$. The distribution of $j$ upon arrival at the first hop is sharply peaked at $j=79$, with tails extending to $j=69$ and 85 . The 

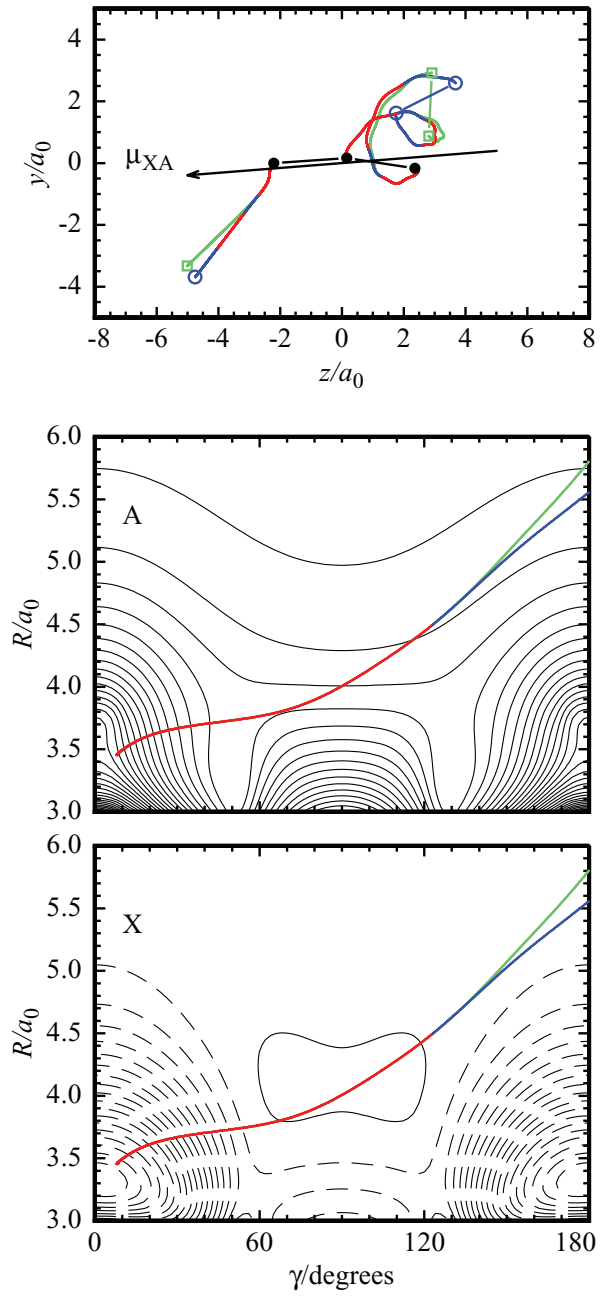

FIG. 6. Behavior of trajectories beginning with the same coordinates and momenta in one- and two-state calculations. In all panels, the one-state trajectory is shown in green. The two-state trajectory is overlaid, in red when it is on the A state and in blue when it is on X. Top panel: atomic position "tracks" plotted in space-fixed coordinates with the $\mathrm{N}_{2} \mathrm{O}$ center of mass at the origin, and the direction of the transition dipole vector, $\boldsymbol{\mu}_{\mathrm{XA}}$. Lower panels: the same trajectories plotted against potential contours of the $\mathrm{X}$ and $\mathrm{A}$ states, computed for $r=2.13 a_{0}$. The contour interval is $0.2 \mathrm{eV}$; contours below the $3.7116 \mathrm{eV}$ dissociation limit are shown dashed.

energy conservation procedure with the democratic prescription resulted in $\Delta j<2$ for $79.2 \%$ of the hops, $\Delta j$ between 3 and 5 for $18.5 \%$, and $\Delta j<8$ for almost all the remainder. The net effect of the energy conservation procedure was to shift the maximum of the $j$ distribution up by roughly one quantum, and slightly extend the high- $j$ tail. The change in $j$ from the dynamics following the first hop was typically two to three times larger than the change at the first hop. We concluded that the discontinuities introduced by the hops, while not negligible, were small enough not to hinder a straightforward interpretation of the trajectories.

Figure 6 shows two versions of one trajectory, started from the same initial coordinates and momenta in either onestate or two-state TSH calculations. This trajectory is representative of those in the high- $j$ tail; in the TSH calculations it ends with $j=84$, while in one-state calculations it ends with $j=73$. In the top panel, which shows the atomic paths, the change in direction of the $\mathrm{O}$ atom and the increasing rota-

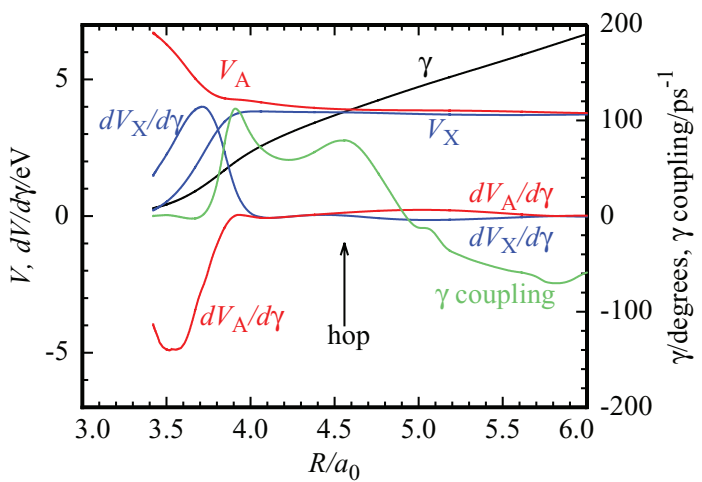

FIG. 7. Behavior of the potentials (in eV), their $\gamma$ derivatives (in $\mathrm{eV} /$ radian), and the nonadiabatic coupling $\dot{\gamma} D_{\gamma}$ as in Eq. (8) of Schmidt et al. (Ref. 14) (in $\mathrm{ps}^{-1}$ ), along a representative trajectory. This trajectory ends with $j=86$ in two-state calculations and 79 in one-state calculations. The first hop (the only dynamically important one) occurs at the position indicated. The energy conservation procedure at that hop causes $\Delta j=+0.82$. In many trajectories, the two $\gamma$ derivatives change more abruptly than this example in the region of $R=3.8 a_{0}$. The derivative behavior for $4.5 a_{0} \leq R \leq 5.8 a_{0}$ is very similar in all trajectories.

tion of the $\mathrm{N}_{2}$ fragment following the hop are apparent. The change in direction of the $\mathrm{O}$ atom away from $\boldsymbol{\mu}_{\mathrm{XA}}$ represents an increase in $\theta_{m}$ and a corresponding decrease in $\beta$. The lower two panels of Figure 6 clarify the cause of the sudden turn. On the $\mathrm{X}$ state surface, once $\gamma>90$, the $\mathrm{O}$ atom feels an attraction toward the terminal $\mathrm{N}$ atom. In effect, the well corresponding to an end-to-end isomerization reaction pulls the $\mathrm{O}$ atom further from its initial direction. The steep fall in $\beta(j)$ beyond the rotational maximum, seen clearly in the experiments at $203.25 \mathrm{~nm}$, is very well reproduced by the TSH calculations.

Another view of the relevant forces is given by Figure 7 , which shows the potentials, their $\gamma$ derivatives, and the nonadiabatic coupling along another representative trajectory. At the beginning of the trajectory, the $\mathrm{X}$ and A state surfaces are widely separated, but as $\gamma$ and $R$ increase they quickly come together. The trajectory crosses the angular well on the A state near $R=3.9 a_{0}$, where the separation between the $\mathrm{X}$ and A state potentials has already decreased to $<1 \mathrm{eV}$. The nonadiabatic coupling first becomes large in the region of the avoided crossing between $\mathrm{X}$ and $\mathrm{A}$ states around $R=3.75 a_{0}$ and $\gamma=50^{\circ}$. Surface hops become possible once the trajectory has passed that point. The behavior of the two angular derivatives, $d V_{\mathrm{X}} / d \gamma$ and $d V_{\mathrm{A}} / d \gamma$, beyond $R=4.5 a_{0}$ shown in Figure 7 represents that of most trajectories we examined: $d V_{\mathrm{X}} / d \gamma<0$ so that trajectories on the $\mathrm{X}$ state feel an additional torque that increases $j$, deflects the $\mathrm{O}$ atom further from its initial departure direction, and decreases $\beta$. On the other hand, $d V_{\mathrm{A}} / d \gamma>0$ in this region, so that trajectories that remain on the A state lose a few quanta of $\mathrm{N}_{2}$ rotation and are deflected back toward higher $\beta$ in this part of the trajectory.

We conclude that the steep fall in $\beta$ in the high- $j$ tail of the rotational distribution is caused directly by nonadiabatic effects, namely, the increased rotational excitation obtained on the $\mathrm{X}$ electronic state in the exit channel beyond $\gamma=90$. This conclusion is consistent with that of Neyer et al., ${ }^{19}$ who stated that "these highest $J$ values are the result of the longer 
range torques in the exit channel of the $\mathrm{N}_{2} \mathrm{O}$ potential" but assigns a particular origin to them and identifies the steep fall as a signature of nonadiabatic behavior.

Kawamata et al. ${ }^{10}$ interpreted their $\beta(j)$ observations on $\mathrm{N}_{2} \mathrm{O}$ in terms of relative contributions of $2^{1} A^{\prime}$ and $1{ }^{1} A^{\prime \prime}$ electronic states to the absorption. They used the expected $\beta=$ -1 for dissociations on $1{ }^{1} A^{\prime \prime}$, and assumed that $\beta=1.8$ for dissociations on $2{ }^{1} A^{\prime}$ in analogy with the high $\beta$ values observed in OCS photodissociation. They concluded that absorption to $2{ }^{1} A^{\prime}$ contributed $54 \%-75 \%$ of the dissociation. Kawamata $e t$ al. recognized that their conclusion depended on the assumed value of $\beta$ for the pure $2^{1} A^{\prime}$ dissociation and discussed the effects of lower assumed values but did not consider values as low as those our trajectories yield for the pure $2{ }^{1} A^{\prime}$ dissociation.

\section{Empirical model}

With detailed trajectories in hand, we are in a position to examine the empirical model described by Demyanenko et al. ${ }^{35}$ in the context of $\mathrm{NO}_{2}$ photodissociation and applied in modified form by Brouard et al. to both OCS and $\mathrm{N}_{2} \mathrm{O}$ photodissociation. ${ }^{36}$ The model, like our trajectory study, uses classical mechanics and assumes zero total angular momentum. It does not attempt to describe the product rotational distribution. Instead, it describes the relation between an observed final rovibrational state and the corresponding value of $\beta$. In our notation, the model evaluates $\beta=2 P_{2}\left(\cos \theta_{m}\right)$, where $\theta_{m}=\phi_{v}-\phi_{\mu}$ gives the angle between the exit direction of the atomic product and the orientation of the transition dipole moment, just as we do. Figure 1 shows the relations among angles from the two descriptions.

Demyanenko et al. were considering $\mathrm{NO}_{2}$ photodissociation in $C_{2 v}$ geometry, for a transition whose transition dipole moment was constrained by symmetry to lie parallel to a line connecting the two $\mathrm{O}$ atoms. They therefore had no need of a parameter describing the orientation of the transition dipole moment. Brouard et al. were considering $A^{\prime} \leftarrow A^{\prime}$ transitions in $C_{s}$ symmetry, for which the transition dipole moment is restricted only to the molecular plane. They therefore included a parameter $\delta$ giving its orientation with respect to the initial $\boldsymbol{R}$ vector. In our notation, $\delta=180^{\circ}-\phi_{\mu}$.

Demyanenko et al. cast their model in terms of a "critical geometry" of the molecule, reached when the Jacobi length $R$ attains the value $R_{C}$ at which the anisotropy of the potential has become negligible and the angular momentum $j$ of the diatomic product has reached its final value. They then resolved the angle between the final $\mathrm{O}$ atom velocity $\boldsymbol{v}_{\mathrm{O}}$ and the initial $\boldsymbol{R}$ vector into a sum of two components. The first, $\Delta \alpha$, describes the rotation of $\boldsymbol{R}$ due to the distortion of the molecule between the initial excitation and the moment the critical geometry is reached. The second, called $\phi$, arises from the tangential velocity of the $\mathrm{O}$ atom in this critical geometry. Since the total angular momentum $J=0$, the rotation $j$ of the product diatomic must be accompanied by a rotation of the $\boldsymbol{R}$ vector, and using an angular momentum argument Demyanenko et al. provided a formula relating $\phi$ to $R_{C}, j$, and the speed of the atomic product. We note that this formula, their
Eq. (8), is valid for any choice of $R_{C}$ long enough that the anisotropy is negligible. Choosing a larger $R_{C}$ simply gives a different partitioning of the exit angle into $\Delta \alpha$ and $\phi$, with larger $R_{C}$ giving larger $\Delta \alpha$ and smaller $\phi$. If the empirical model is successful, the dynamics will be such that $\Delta \alpha$ is effectively constant for some choice of $R_{C}$ and all the variation in $\beta(j)$ will come from variations in $\phi$. Demyanenko et al. used $R_{C}$ and $\alpha_{C}=\Delta \alpha+\alpha_{i}$, where the known $\alpha_{i}$ gives the initial angle between $\boldsymbol{R}$ and the $a$ principal axis, as adjustable parameters to fit $\beta(j)$ curves for $\mathrm{NO}_{2}$ dissociation at several wavelengths.

The natural extension of the model for the purposes of Brouard et al. would have taken the form $\beta=2 P_{2}(\cos (\phi$ $+\Delta \alpha-\delta)$ ), using Eq. (8) of Demyanenko et al. to relate $\phi$ to $j$. However, the $\beta(j)$ data constrain only the difference between $\Delta \alpha$ and $\delta$. Brouard $e t a l$. effectively assumed $\Delta \alpha$ $=0$; that is, they assumed that the $\boldsymbol{R}$ vector was still in its original orientation when the product $j$ was established. They then used $\beta=2 P_{2}(\cos (\delta-\phi))$, treating $R_{C}$ and $\delta$ as their fitting parameters and relating $\phi$ (which they renamed $\alpha^{\prime}$ ) to $j$ through $R_{C}$ as described above. For $\mathrm{N}_{2} \mathrm{O}$ photodissociation at $203 \mathrm{~nm}$, they fit to the experimental data and found $\delta=-20^{\circ}$ at $R_{C}=2.8 \AA$.

With our trajectories, we can test the performance of the model and of the $\Delta \alpha=0$ assumption of Brouard et al. Figure 4 shows the $\delta$ and $\alpha^{\prime}$ values from their fits, converted to our axis system for comparison, as solid lines. (The plotted values are $180-\delta$ and $180-\alpha^{\prime}$.) The curves are clearly displaced from our computed $\phi_{\mu}$ and $\phi_{v}$. However, the difference between the $\delta$ and $\alpha^{\prime}$ curves at each $j$ does correspond fairly accurately to the corresponding difference between $\phi_{\mu}$ and $\phi_{v}$, as would be expected since this difference controls $\beta$ and both treatments give $\beta$ in agreement with the experiment.

Brouard et al. fitted $R_{C}=2.8 \AA$. We examined a randomly selected set of 40 trajectories and determined the value of $\left|\phi_{R}\right|$, the rotation of the $\boldsymbol{R}$ vector from its original orientation, at the time that $R$ reached $2.8 \AA$. The average value was $23.5^{\circ}$. This value represents an independently determined estimate of $\Delta \alpha$ for the empirical model. If we shift both the $\delta$ and $\alpha^{\prime}$ curves in Figure 4 down by that amount, as shown by the dashed lines, we find that they agree reasonably well with the curves determined from our trajectories, especially in the central part of the rotational distribution. The values of $\beta$ predicted by the model are unaffected by this shift.

The model assumes that the transition dipole orientation and the distortion of the molecule before the torques vanish are the same for all dissociations, so that the change in $\beta$ across the rotational distribution arises entirely from the change in tangential velocity with $j$. We found that in the central part of the rotational distribution, this assumption was not accurate; our $\phi_{v}$ curve is approximately flat, and our $\phi_{\mu}$ rising, for $65 \leq j \leq 80$. The empirical model cannot be expected to capture such dynamical details. Nonetheless its overall success, once an appropriate value of $\Delta \alpha$ is available, is quite good. The estimate of the orientation of the transition dipole moment obtained by Brouard et al. ${ }^{36} \delta=-20^{\circ}$, is in error because of the incorrect value assumed for $\Delta \alpha$. We agree, however, with the conclusion of Brouard et al. that the observed $\beta$ values are caused principally by nonaxial recoil. We also 
agree that the decrease in $\beta$ at high $j$ is caused by an increase in nonaxial recoil associated with the higher $j$ values. Since $\beta$ decreases with $j$, increased nonaxial recoil must be moving the final velocity vectors away from the initial transition dipole moment direction.

\section{SUMMARY}

Trajectory surface hopping calculations, based on excitation purely to the $2^{1} A^{\prime}$ state of $\mathrm{N}_{2} \mathrm{O}$ but including nonadiabatic coupling to the ground state, yield photodissociation anisotropy parameters $\beta$ in good agreement with experiment. The observed $\beta$ are substantially lower than the limiting value of +2 ; nonaxial recoil caused by strong bending forces during the dissociation is the main cause.

In the main part of the distribution and the low- $j$ tail, the fragments remain on the excited state surface during the whole dissociation. An unusual dynamical correlation influences the $\beta$ values for the $203 \mathrm{~nm}$ dissociation; for $65 \leq j$ $\leq 80$, the average outgoing fragment angle changes very little but excited molecules with longer $\mathrm{N}-\mathrm{N}$ bonds tend to have higher final $j$ and to have transition dipole moments that lie closer to the $\mathrm{N}_{2} \mathrm{O}$ principal axis. This combination of factors causes a decrease in $\beta$ with increasing $j$.

The high- $j$ tail of the rotational distribution at both 203 and $193 \mathrm{~nm}$ is caused by nonadiabatic coupling to the ground state late in the dissociation. Additional torque present on the ground state surface, associated with the end-to-end isomerization pathway, increases the rotational energy and the associated nonaxial recoil of the fragments and causes a steep decrease in $\beta$.

\section{ACKNOWLEDGMENTS}

The authors thank Nicholas Kamar for his initial adaptation of the $\beta$-calculation program to $\mathrm{N}_{2} \mathrm{O}$ and Mark Brouard for comments on the manuscript. G.C.M. is grateful to the Max-Planck-Institut für Dynamik und Selbstorganisation for hospitality and financial support.

${ }^{1}$ D. G. Hopper, J. Chem. Phys. 80, 4290 (1984).

${ }^{2}$ A. Brown, P. Jimeno, and G. G. Balint-Kurti, J. Phys. Chem. A 103, 11089 (1999).

${ }^{3}$ M. N. Daud, G. G. Balint-Kurti, and A. Brown, J. Chem. Phys. 122, 054305 (2005).

${ }^{4}$ S. Nanbu and M. S. Johnson, J. Phys. Chem. A 108, 8905 (2004).

${ }^{5}$ R. Schinke, J. Chem. Phys. 134, 064313 (2011).
${ }^{6}$ T. F. Hanisco and A. C. Kummel, J. Phys. Chem. 97, 7242 (1993).

${ }^{7}$ D. W. Neyer, A. J. R. Heck, D. W. Chandler, J. M. Teule, and M. H. M. Janssen, J. Phys. Chem. A 103, 10388 (1999).

${ }^{8}$ J. M. Teule, G. C. Groenenboom, D. W. Neyer, D. W. Chandler, and M. H. M. Janssen, Chem. Phys. Lett. 320, 177 (2000).

${ }^{9}$ T. Nishide and T. Suzuki, J. Phys. Chem. A 108, 7863 (2004).

${ }^{10}$ H. Kawamata, H. Kohguchi, T. Nishide, and T. Suzuki, J. Chem. Phys. 125, 133312 (2006).

${ }^{11}$ R. Schinke, J. Suarez, and S. C. Farantos, J. Chem. Phys. 133, 091103 (2010).

${ }^{12}$ R. Schinke, "Photodissociation of $\mathrm{N}_{2} \mathrm{O}$ : Temperature dependence," Chem. Phys. (in press).

${ }^{13}$ J. A. Schmidt, M. S. Johnson, and R. Schinke, Atmos. Chem. Phys. 11, 8965 (2011)

${ }^{14}$ J. A. Schmidt, M. S. Johnson, U. Lorenz, G. C. McBane, and R. Schinke, J. Chem. Phys. 135, 024311 (2011).

${ }^{15}$ J. C. Tully, J. Chem. Phys. 93, 1061 (1990).

${ }^{16}$ P. Felder, B. M. Haas, and J. R. Huber, Chem. Phys. Lett. 186, 177 (1991).

${ }^{17}$ L. L. Springsteen, S. Satyapal, Y. Matsumi, L. M. Dobeck, and P. L. Houston, J. Phys. Chem. 97, 7239 (1993).

${ }^{18}$ M. Ahmed, E. R. Wouters, D. S. Peterka, O. S. Vasyutinskii, and A. G. Suits, Faraday Discuss. 113, 425 (1999).

${ }^{19}$ D. W. Neyer, A. J. R. Heck, and D. W. Chandler, J. Chem. Phys. 110, 3411 (1999).

${ }^{20}$ N. R. Cheong, H. S. Park, S. H. Nam, S. K. Shin, S. G. Cho, H. W. Lee, J. K. Song, and S. M. Park, Bull. Korean Chem. Soc. 30, 2661 (2009).

${ }^{21}$ T. Suzuki, H. Katayanagi, Y. X. Mo, and K. Tonokura, Chem. Phys. Lett. 256, 90 (1996).

${ }^{22}$ A. G. Smolin, O. S. Vasyutinskii, E. R. Wouters, and A. G. Suits, J. Chem. Phys. 121, 6759 (2004).

${ }^{23}$ T. H. Dunning, Jr., J. Chem. Phys. 90, 1007 (1989).

${ }^{24}$ H.-J. Werner and P. J. Knowles, J. Chem. Phys. 82, 5053 (1985).

${ }^{25}$ P. J. Knowles and H.-J. Werner, Chem. Phys. Lett. 115, 259 (1985).

${ }^{26}$ H.-J. Werner and P. J. Knowles, J. Chem. Phys. 89, 5803 (1988).

${ }^{27}$ P. J. Knowles and H.-J. Werner, Chem. Phys. Lett. 145, 514 (1988).

${ }^{28}$ H.-J. Werner, P. J. Knowles, R. Lindh, M. Schütz, P. Celani et al., MOLPRO, version 2002.6, a package of ab initio programs, 2003, see http://www.molpro.net.

${ }^{29}$ R. Schinke, Photodissociation Dynamics (Cambridge University Press, Cambridge, 1993).

${ }^{30}$ R. Schinke and G. C. McBane, J. Chem. Phys. 132, 044305 (2010).

${ }^{31}$ N. C. Blais and D. G. Truhlar, J. Chem. Phys. 79, 1334 (1983).

${ }^{32}$ M. D. Hack, A. W. Jasper, Y. L. Volobuev, D. W. Schwenke, and D. G. Truhlar, J. Phys. Chem. A 103, 6309 (1999).

${ }^{33}$ R. N. Zare, Angular Momentum: Understanding Spatial Aspects in Chemistry and Physics (Wiley, New York, 1988).

${ }^{34}$ G. C. McBane, L. T. Nguyen, and R. Schinke, J. Chem. Phys. 133, 144312 (2010).

${ }^{35}$ A. V. Demyanenko, V. Dribinski, H. Reisler, H. Meyer, and C. X.W. Qian, J. Chem. Phys. 111, 7383 (1999).

${ }^{36}$ M. Brouard, A. V. Green, F. Quadrini, and C. Vallance, J. Chem. Phys. 127, 084304 (2007)

${ }^{37}$ N. Shafer, K. Tonokura, Y. Matsumi, S. Tasaki, and M. Kawasaki, J. Chem. Phys. 95, 6218 (1991).

${ }^{38}$ T. Suzuki, H. Katayanagi, S. Nanbu, and M. Aoyagi, J. Chem. Phys. 109, 5778 (1998). 